Check for updates

Cite this: Phys. Chem. Chem. Phys. 2019, 21, 16989

Received 8th April 2019 Accepted 15th July 2019 DOI: $10.1039 / c 9 c p 01953 g$

rsc.li/pccp

\title{
Influence of polar co-solutes and salt on the hydration of lipid membranes $\dagger$
}

\author{
Amanuel Wolde-Kidan, (D) ${ }^{a}$ Quoc Dat Pham, (D) ${ }^{b}$ Alexander Schlaich, (D) ${ }^{c}$ \\ Philip Loche, (D) a Emma Sparr, (D) ${ }^{b}$ Roland R. Netz ${ }^{a}$ and Emanuel Schneck (D) *d
}

\begin{abstract}
The influence of the co-solutes TMAO, urea, and $\mathrm{NaCl}$ on the hydration repulsion between lipid membranes is investigated in a combined experimental/simulation approach. Pressure-hydration curves obtained via sorption experiments reveal that the repulsion significantly increases when the membranes are loaded with co-solutes, most strongly for TMAO. As a result, the co-solutes retain additional water molecules and therefore provide membranes with a fluid and more physiological environment. The experimental data are quantitatively reproduced in complementary solvent-explicit atomistic molecular dynamics simulations, which yield the chemical potential of water. Simulation analysis reveals that the additional repulsion arises from the osmotic pressure generated by the co-solutes, an effect which is maximal for TMAO, due to its unfavorable interactions with the lipid headgroup layer and its extraordinarily high osmotic coefficient.
\end{abstract}

\section{Introduction}

Lipid membranes in water experience a strong mutual repulsion at separations below 2-3 nm. ${ }^{1,2}$ This so-called hydration repulsion plays an essential biological role as it creates a barrier against close membrane contacts and thereby suppresses uncontrolled membrane adhesion and fusion. ${ }^{3}$ In addition, it provides lipid membrane systems with the fluid environment required for vital functioning. Under dry conditions, however, dehydration due to osmotic stress can affect lipid self-assembly with far-reaching consequences. For example, dehydration-induced phase transitions, which shift the membrane state from fluid to gel-like, ${ }^{4,5}$ have been associated with altered barrier functions of the human stratum corneum (SC), the outermost layer of the skin. ${ }^{6}$ The SC is only a few micrometers thick and formed by dead corneocyte cells embedded in a lipid multilamellar matrix, where the bilayers are arranged parallel to the skin surface. ${ }^{7}$ It exhibits very low permeability for hydrophilic and hydrophobic molecules. ${ }^{8}$

In nature, protection against osmotic stress is commonly achieved by the release of small polar co-solutes with low vapor pressure, sometimes referred to as osmolytes. ${ }^{9,10}$ These molecules serve to retain the physiological, fluid state of membrane systems in low humidity conditions. This strategy is taken by

\footnotetext{
${ }^{a}$ Fachbereich Physik, Freie Universität Berlin, Arnimallee 14, 14195 Berlin, Germany

${ }^{b}$ Division of Physical Chemistry, Chemistry Department, Lund University, P.O. Box 124, 22100 Lund, Sweden

${ }^{c}$ Université Grenoble Alpes, CNRS, LIPhy, 38000 Grenoble, France

${ }^{d}$ Biomaterials Department, Max Planck Institute of Colloids and Interfaces, 14476 Potsdam, Germany. E-mail: schneck@mpikg.mpg.de

$\dagger$ Electronic supplementary information (ESI) available. See DOI: 10.1039/c9cp01953g
}

algae and higher plants, which produce small carbohydrates and polyols, like glycerol. ${ }^{11,12}$ Similarly, insects and higher animals rely on glycerol, urea, and trimethylamine- $N$-oxide (TMAO), among others. ${ }^{13,14}$ The human skin contains a mixture of small polar compounds that is referred to as the "natural moisturizing factor" (NMF), comprising free amino acids, derivatives thereof, lactic acid, urea, and glycerol. ${ }^{15,16}$ The manifestation of NMF components in the SC is well documented, and their presence is considered crucial to maintain softness and pliability. ${ }^{16,17}$ In fact, defective skin conditions and certain skin diseases, for example winter xerosis and atopic dermatitis, are associated with decreased NMF levels in the SC. ${ }^{18,19}$

Previous studies on urea, glycerol, and small carbohydrates have shown that these molecules act as non-volatile substitutes of water molecules, thereby protecting self-assembled structures that are otherwise only present in more hydrated conditions. ${ }^{20-22}$ More recently, it was shown that TMAO, in contrast to urea, exhibits unfavorable interactions with lipid headgroups and is therefore preferentially expelled from membrane multilayers via precipitation at very dry conditions ${ }^{23}$ or by partitioning into the excess aqueous medium when present. ${ }^{24}$ In a wide range of dehydrating osmotic pressures, however, both urea and TMAO remain confined between the membrane surfaces. ${ }^{23}$

In the present work, we investigate the influence of cosolutes on the hydration repulsion between lipid membranes, the interfacial force that ultimately governs the hydration level of membrane systems subject to osmotic stress. ${ }^{1}$ Pressurehydration curves obtained via sorption calorimetry and sorption balance experiments of phospholipid multilayers containing defined amounts of urea, TMAO, and of the salt $\mathrm{NaCl}$ reveal that the repulsion can be dramatically enhanced, depending on 
A

DMPC:

$\mathrm{R}_{1}=\mathrm{R}_{2}=\mathrm{C} 14: 0$

$\left(\mathrm{C}_{14} \mathrm{H}_{27} \mathrm{O}\right.$ myristic acid $)$

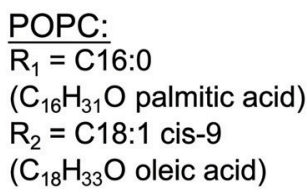

$\left(\mathrm{C}_{18} \mathrm{H}_{33} \mathrm{O}\right.$ oleic acid)

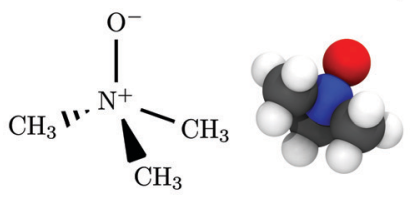

TMAO

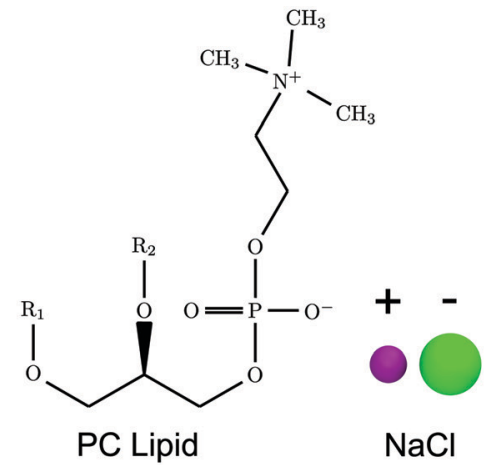

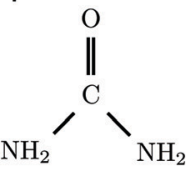

Urea
B

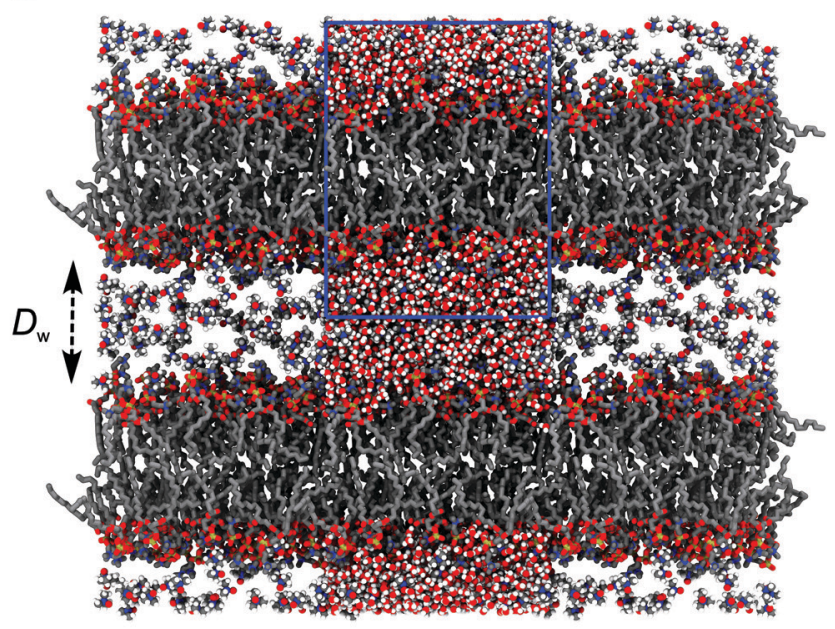

Fig. 1 (A) Chemical structures of the studied lipids and co-solutes. (B) Snapshot from simulations of DMPC bilayers loaded with 10 wt\% TMAO at a hydration level of $n_{\mathrm{w}}=25$ water molecules per lipid, corresponding to a water layer thickness of $D_{\mathrm{w}} \approx 2.5 \mathrm{~nm}$. The simulation box is indicated with a blue rectangle. Water is only shown in the central periodic images.

the chemistry of the co-solute (for chemical structures see Fig. 1A). The effect of TMAO is 2-3 times stronger than that of urea or NaCl. The pressure-hydration curves are quantitatively reproduced in solvent-explicit atomistic molecular dynamics (MD) simulations, which precisely yield the chemical potential of water $^{25,26}$ (for a simulation snapshot see Fig. 1B). Detailed analysis of the simulation results provides the physical explanation for the observed repulsion enhancement: additional repulsion arises from the osmotic pressure generated by the cosolutes, an effect which is amplified for TMAO, due to its unfavorable interactions with the membrane surfaces and its extraordinarily high osmotic coefficient. For high enough TMAO weight fractions, the thermodynamics of membrane dehydration is dominated by the dehydration of the confined TMAO solution.

While the fluidizing effect of small polar co-solutes has so far been mainly attributed to their ability to substitute water molecules, ${ }^{21,22}$ the enhancement of the hydration repulsion identified and explained in the present work demonstrates that co-solutes can additionally have a moisturizing effect in the literal sense: at dry conditions, i.e., depressed water chemical potential, they retain additional water molecules and therefore provide membranes with a fluid and more physiological environment.

\section{Materials and methods}

\subsection{Sample preparation}

The phospholipids dimyristoylphosphatidylcholine (DMPC) and palmitoyl-oleoyl-phosphatidylcholine (POPC) were purchased from Avanti Polar Lipids, Inc. (Alabaster, AL). Urea and TMAO were obtained from Sigma-Aldrich and $\mathrm{NaCl}$ from VWR. The samples were prepared so that the co-solute-lipid mass fraction (of urea, TMAO or NaCl $)$ in the dry samples, $\Psi_{\text {cosol }}^{\text {lip }}=m_{\text {cosol }}\left(m_{\text {cosol }}+m_{\text {lip }}\right)$, was fixed while the water content in terms of the number of water molecules per lipid $n_{\mathrm{w}}=N_{\mathrm{w}} / N_{\text {lip }}$ is variable. The samples were mixed together by the use of a mortar and pestle to produce a fine powder, which was then subjected to drying under vacuum with a $3 \AA$ molecular sieve for at least 24 hours. According to earlier reports, ${ }^{27,28}$ this drying procedure removes all water from the lipid sample. The dry samples were then loaded into the measurement device under a stream of dry $\mathrm{N}_{2}$.

\subsection{Sorption calorimetry measurements}

A double twin isothermal microcalorimeter was used to study the thermodynamics of the water vapor sorption. ${ }^{29}$ The instrument involves a two-chamber calorimetric cell, with a sorption chamber that contains the dry sample and a vaporization chamber in which liquid water is injected to start the sorption experiment. The chambers are connected by a tube. Water vaporizes in the vaporization chamber and diffuses through the tube to the sorption chamber where it is absorbed by the sample. The calorimetric cell is inserted into a double twin isothermal microcalorimeter that separately measures thermal powers released or absorbed in the two chambers. The data for each experiment were recorded for $c a .14$ days at $T=27^{\circ} \mathrm{C}$. The experimental setup can be considered a continuous titration of an initially dry sample with water vapor. ${ }^{29}$ Using this method, one can simultaneously monitor the water uptake in terms of $n_{\mathrm{w}}$, the partial enthalpy of water, and the relative humidity $h_{\text {rel }}$, which is then converted into the water chemical potential as $\Delta \mu=-R T \ln \left(h_{\text {rel }} / 100\right)$, where $R$ is the gas constant.

In addition, osmotic coefficients $\Phi$ of TMAO, urea and $\mathrm{NaCl}$ can be determined from the water chemical potential in aqueous solutions at various co-solute concentrations, using the following equation $^{30}$

$$
\Phi\left(x_{\mathrm{w}}\right)=\frac{\Delta \mu}{\Delta \mu_{\mathrm{is}}}=\frac{\Delta \mu}{R T \ln \left(x_{\mathrm{w}}\right)},
$$

where $\Delta \mu_{\text {is }}$ is the water chemical potential in an ideal solution which solely depends on the water mole fraction $x_{\mathrm{w}}$ and $\Delta \mu$ 
denotes the real water chemical potential. The mole fraction of water can also be expressed in terms of the molal solute concentration $b$ as $x_{\mathrm{w}}(b)=1 /\left(1+\nu M_{\mathrm{w}} b\right)$, where $M_{\mathrm{w}}$ is the molar mass of water and $\nu$ is the ideal van't Hoff factor $\left(\nu_{\mathrm{NaCl}}=2\right.$, $\nu_{\text {TMAO }}=\nu_{\text {urea }}=1$ ).

\subsection{Sorption microbalance measurements}

Sorption balance measurements were performed using an Aquadyne DVS microbalance device from Quantachrome Instruments. The dry samples were placed on pans in the device and exposed to a stream of nitrogen at controlled relative humidity $h_{\text {rel }}$. The balance measured the mass gain or loss over time with a weight reading every $5 \mathrm{~s}$. Experiments started with drying steps, where $h_{\text {rel }}$ was brought as closely as possible to zero for $240-5000 \mathrm{~min}$ at $40{ }^{\circ} \mathrm{C}$ and then for $180-5000 \mathrm{~min}$ at $27{ }^{\circ} \mathrm{C}$. After the drying, the experiments continued subsequently with a ramp of $h_{\text {rel }}$ steps at $T=27{ }^{\circ} \mathrm{C}$, where each step was performed for $180-5000 \mathrm{~min}$. The condition for the next step to be engaged after the minimum step duration was that the weight change per min was less than $0.0001 \%$ of the starting mass. The total weight of water and dry sample at each $h_{\text {rel }}$ step was obtained from the final plateau values of the weight recorded after each change in $h_{\text {rel }}$ and immediately before the next $h_{\text {rel }}$ step. Sorption isotherms were constructed based on the water content as a function of $h_{\text {rel }}$.

\subsection{Simulations}

Using the GROMACS 2016.4 package, ${ }^{31}$ planar lipid bilayers with $N_{\text {lip }}=72$ lipid molecules ( 36 in each leaflet) were simulated at various hydration levels and with various co-solute contents. For a simulation snapshot see Fig. 1B. Periodic boundary conditions were imposed in all directions, effectively representing infinite stacks of hydrated lipid bilayers. Unless stated otherwise, the Berger lipid forcefield ${ }^{32}$ was employed in combination with SPC/E water ${ }^{33}$ and thermodynamically optimized forcefields for TMAO, ${ }^{34}$ urea $^{35}$ and $\mathrm{NaCl}^{36}$ In order to investigate forcefield effects, selected simulations were repeated with the CHARMM36 forcefield for lipids and ions ${ }^{37,38}$ in combination with the TIP3P water model $^{39,40}$ (see Section 3.3.5). Simulations were run in the $N p T$-ensemble at $T=300 \mathrm{~K}$ and atmospheric pressure of $p=1$ bar. Temperature was controlled using the velocity re-scaling thermostat ${ }^{41}$ with a time constant of $\tau_{\mathrm{T}}=0.5 \mathrm{ps}$, while for the pressure the Berendsen barostat ${ }^{42}$ was used with semi-isotropic pressure coupling with a time constant of $\tau_{\mathrm{p}}=1 \mathrm{ps}$ and a compressibility of $\kappa=4.5 \times 10^{-5} \mathrm{bar}^{-1}$. The timestep was $\Delta t=$ 2 fs. Charge interactions were modeled using the particle-meshEwald (PME) method $^{43}$ and van der Waals interactions were described by Lennard-Jones potentials shifted to zero at the cutoff. For both, electrostatics and van der Waals interactions, a cut-off radius of $r_{\mathrm{c}}=0.9 \mathrm{~nm}$ was used. Starting configurations for the different hydration levels were generated by step-wise dehydration of a highly hydrated bilayer $\left(n_{\mathrm{w}}=25\right)$ and subsequent equilibration for $10 \mathrm{~ns}$. All data points represent averages over five independently dehydrated systems. For density profiles and observables derived thereof data were gathered from $10 \mathrm{~ns}$ production runs of each of the five systems, amounting to a total of $50 \mathrm{~ns}$.
The repulsive pressure $\Pi$ was obtained from the shift $\Delta \mu$ in the water chemical potential according to eqn (2). To determine $\mu=\mu^{\text {ex }}+\mu^{\text {id }}$, its excess and ideal contributions, $\mu^{\text {ex }}$ and $\mu^{\text {id }}=k_{\mathrm{B}} T \ln \left(\rho_{\mathrm{w}} \Lambda^{3} N_{\mathrm{A}} / M_{\mathrm{w}}\right)$, were measured independently, where $\rho_{\mathrm{w}}$ is the water density, $\Lambda \approx 0.0237 \mathrm{~nm}$ is the thermal wavelength of water at $T=300 \mathrm{~K}$ and $N_{\mathrm{A}}$ is Avogadro's number. While $\mu$ by definition is constant over the simulation volume in thermal equilibrium, $\mu^{\text {ex }}$ and $\mu^{\text {id }}$ are not. Due to the inhomogeneous water distribution perpendicular to the membrane surface, $\mu^{\mathrm{ex}}(z)$ and $\mu^{\mathrm{id}}(z)$ via $\rho_{\mathrm{w}}(z)$ are functions of the perpendicular coordinate, $z$. As a consequence, $\mu^{\mathrm{ex}}$ and $\mu^{\text {id }}$ have to be evaluated at the same $z$ position in the simulation box to determine $\mu$. While $\mu^{\text {id }}$ trivially follows from $\rho_{\mathrm{w}}, \mu^{\text {ex }}$ was determined via a computationally efficient combination of two approaches, the test particle insertion ${ }^{44}$ for the LennardJones contribution and the thermodynamic integration with multi-state Bennet acceptance ratio analysis ${ }^{45}$ for the Coulomb contribution. The thermodynamic integration involved $18 \lambda$-steps of at least $10 \mathrm{~ns}$ duration each, amounting to a total simulation time of at least $5 \times 18 \times 10 \mathrm{~ns}=900 \mathrm{~ns}$ per data point, or $7.2 \mu$ s per pressure-distance curve. The water chemical potential of the bulk reference $\mu_{0}$ was approximated by averaging values obtained at the three largest hydration levels $\left(n_{\mathrm{w}}=19,22\right.$, and 25).

Interaction free energies were computed by integrating the change in the water chemical potential as $\Delta G\left(n_{\mathrm{w}}\right)=$ $\int_{n_{\mathrm{w}}}^{\infty} \Delta \mu\left(n_{\mathrm{w}}{ }^{\prime}\right) \mathrm{d} n_{\mathrm{w}}{ }^{\prime}$. The enthalpic contribution was computed from the system's total enthalpy as $\Delta H\left(n_{\mathrm{w}}\right)=H\left(n_{\mathrm{w}}\right)-\left(H\left(n_{\mathrm{w}}^{\max }\right)-\left(n_{\mathrm{w}}^{\max }-\right.\right.$ $\left.\left.n_{\mathrm{w}}\right) \cdot \mathrm{d} H_{\mathrm{w}} / \mathrm{d} N_{\mathrm{w}}\right)$, where $n_{\mathrm{w}}^{\max }=25$. From independent simulations of bulk water we determined $\mathrm{d} H_{\mathrm{w}} / \mathrm{d} N_{\mathrm{w}}=-38.67 \mathrm{~kJ} \mathrm{~mol}^{-1}$ (see Fig. $\mathrm{S} 1$ in the ESI $\dagger$ ).

Osmotic coefficients were determined by measuring the change in water chemical potential $\Delta \mu$ in simulations of bulk co-solute solutions at various concentrations. The corresponding values for the osmotic coefficients $\Phi$ were then calculated using eqn (1).

\section{Results and discussion}

TMAO and urea are commonly-studied co-solutes, due to their abilities to stabilize and destabilize, respectively, the native fold of proteins. ${ }^{46}$ In phospholipid bilayer systems, TMAO has been shown to be repelled from the lipid headgroup region, while urea has a slight affinity for the bilayer surface. ${ }^{23}$ Here, the effect of TMAO and urea as well as $\mathrm{NaCl}$ on the hydration of phospholipid multilayers is investigated. Two lipid species, one with saturated chains and one with partially unsaturated chains, dimyristoyl-phosphatidylcholine (DMPC) and palmitoyl-oleoylphosphatidylcholine (POPC), respectively, are studied. First, the experimental data obtained via sorption calorimetry and sorption balance are presented. This is followed by a description of the simulation results and their comparison with the experimental data. Finally, the underlying mechanisms identified through simulation analysis are discussed. 


\subsection{Co-solute influence on the hydration repulsion: experiments}

Fig. 2 shows the relationships between the dehydrating osmotic pressure $\Pi$ and the hydration level $n_{\mathrm{w}}$ as obtained in sorption experiments for samples composed of POPC lipids with varying amounts of co-solutes (data for DMPC lipids are shown in Fig. S3 in the ESI $\dagger$ ). The dehydrating osmotic pressure follows from the shift in the water chemical potential, $\Delta \mu$ (see Methods section), as

$$
\Pi=-\frac{\Delta \mu}{v_{\mathrm{w}}},
$$

where $v_{\mathrm{w}}$ is the partial molecular volume of water. ${ }^{29}$ In the absence of co-solutes, the curves exhibit an approximately exponential pressure decay with increasing hydration, i.e., with increasing surface separation (see dashed line in Fig. 2C). The latter is defined in terms of an equivalent water layer thickness,

$$
D_{\mathrm{w}}=2 n_{\mathrm{w}} v_{\mathrm{w}}^{0} / A_{\text {lip }}
$$

where $v_{\mathrm{w}}^{0}$ is the volume per water molecule in bulk and $A_{\text {lip }}$ the average area per lipid (see further below). The observed exponential decay in the repulsive pressure reflects the wellknown hydration repulsion between PC lipid membranes., ${ }^{1,47}$ The fitted decay lengths in the absence of co-solute are $\lambda_{\mathrm{DMPC}}^{n_{\mathrm{W}}} \approx 2.3$ and $\lambda_{\mathrm{POPC}}^{n_{\mathrm{w}}} \approx 3.1$ in units of $n_{\mathrm{w}}$. For areas per lipid of $A_{\text {lip }} \approx 0.60-0.65 \mathrm{~nm}^{2}$ (see Fig. 4), this corresponds to decay lengths of $\lambda_{\mathrm{DMPC}} \approx 0.21 \mathrm{~nm}$ and $\lambda_{\mathrm{POPC}} \approx 0.29 \mathrm{~nm}$, in agreement with the literature. ${ }^{47,48}$ The addition of co-solutes significantly increases both strength and range of the repulsion. The increase in the hydration level $n_{\mathrm{w}}$ for a fixed osmotic pressure upon co-solute addition clearly demonstrates that the presence of co-solutes leads to the retention of additional water molecules for a given humidity level. The strength of this effect is monotonic in the added co-solute mass fraction, as seen for TMAO in panel A, but it is not simply proportional to the cosolute concentration. Instead, the repulsion is amplified more strongly for TMAO, although the added mass fraction corresponds to a lower co-solute concentration compared to the other co-solutes with a smaller molar mass. The results in Fig. 2 demonstrate that co-solutes have a pronounced influence on the hydration repulsion between lipid membranes, which is however strongly dependent on the co-solute chemistry. The underlying physical mechanisms are discussed in the following on the basis of the results obtained with complementary MD simulations.

\subsection{Comparison between experiments and simulations}

Over the last few years we have demonstrated that dehydrating osmotic pressures in atomistic MD simulations can be determined via precise measurements of the water chemical potential $\mu$ as a function of the hydration level..$^{25,26}$ In analogy to the experimental procedure, the osmotic pressure follows from $\Delta \mu=\mu-\mu_{0}$ according to eqn (2). In the simulations the reference chemical potential in pure bulk water is $\mu_{0}=-48.3 \mathrm{~kJ} \mathrm{~mol}^{-1}$ and the partial molecular volume of water, $v_{\mathrm{w}}$, is found to be approximately equal to $v_{\mathrm{w}}^{0} \approx 0.0304 \mathrm{~nm}^{3}$ for all hydration levels (see $\mathrm{ESI} \dagger$ ). The main simulation results are reported for the Berger lipid forcefield, which is known to quantitatively reproduce pressure-distance curves between lipid bilayers, ${ }^{26}$ and which uses the SPC/E water model, for which also the co-solute forcefields have been optimized. ${ }^{34-36}$

As seen in Fig. 2, the pressure vs. hydration data obtained in the simulations are in near-quantitative agreement with the corresponding experimental data on POPC. While the agreement between simulations and experiments for lipid membranes in pure water has been demonstrated earlier, ${ }^{26,47}$ we here show that the simulations also accurately reproduce the effect of added TMAO and urea. This agreement lends credibility to the employed forcefields and simulation methodology and motivates further analysis of the simulation trajectories as discussed below. In the case of $\mathrm{NaCl}$ the simulations significantly underestimate the added repulsion, which must likely be
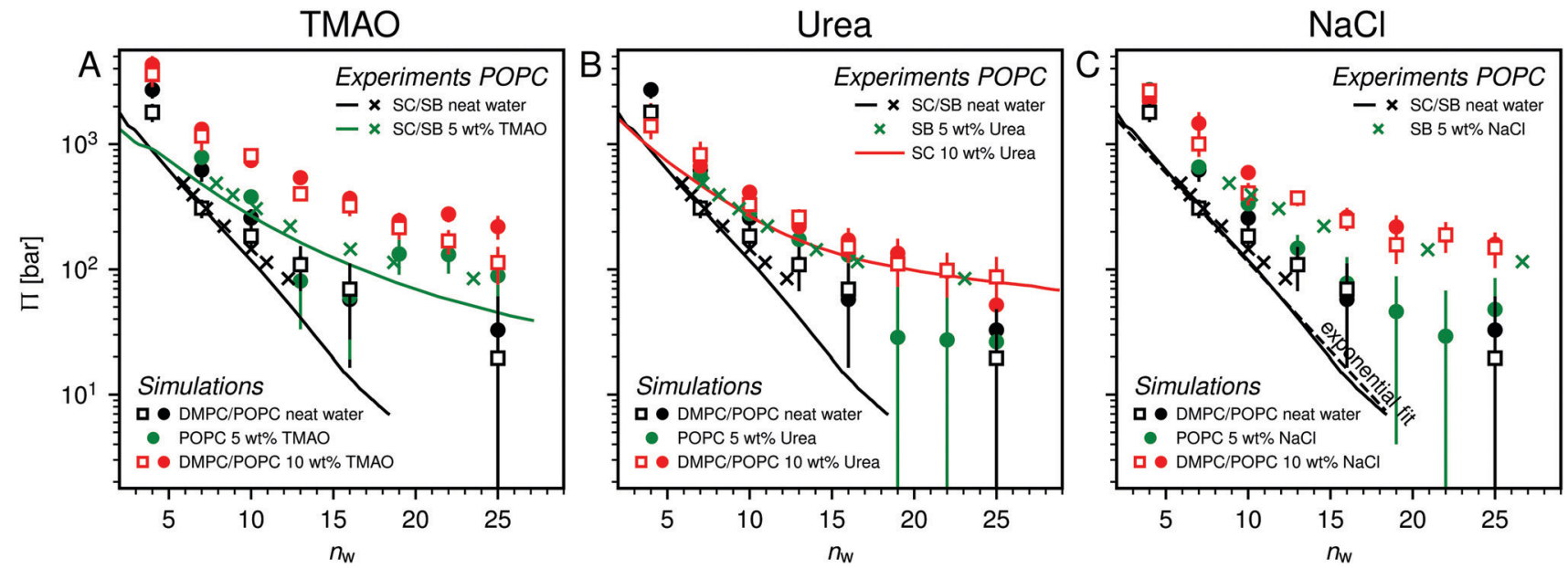

Fig. 2 Pressure-hydration curves $\Pi\left(n_{\mathrm{w}}\right)$ of POPC membranes with and without added TMAO (A), urea (B), and $\mathrm{NaCl}(\mathrm{C})$, as obtained in sorption balance (SB - crosses) and sorption calorimetry (SC - solid lines) experiments and simulations. The figure also contains simulation data for DMPC. The dashed line in panel (C) indicates an exponential fit to the sorption calorimetry data in the absence of co-solutes, with decay length $\lambda_{\mathrm{POPC}}=0.29 \mathrm{~nm}$ (see main text). 
attributed to shortcomings in the forcefield regarding the interaction of $\mathrm{Na}^{+}$with the membrane surfaces (see Section 3.3.5). The simulation data for DMPC are also shown in Fig. 2. They are very similar to the ones obtained with POPC and, as shown in the ESI, $\dagger$ agree with the experiments, but only within the limits of their comparability. Namely, DMPC at low hydration levels undergoes a phase transition from fluid to gel-like, which is not captured on the time scale of the MD simulations. ${ }^{5}$ Moreover, phase separation of co-solutes from the lipid phase, which was reported experimentally earlier for low hydration levels, ${ }^{23}$ by construction cannot occur in the MD simulations which involve only a single bilayer. Generally, it should be noted that simulations of POPC or DMPC multilayers loaded with as much as $10 \mathrm{wt} \%$ co-solutes for the same reasons do not always have an experimental counterpart, especially at very low hydration. They are nonetheless helpful to elucidate the physical mechanisms underlying the experimentally observed effects.

\subsection{Simulation analysis}

3.3.1 Membrane-co-solute interactions. It was previously shown that the partitioning of co-solutes within multilamellar membrane systems is sensitive to the co-solutes' preferential interactions with the membrane surfaces. ${ }^{23,24}$ It is therefore likely that co-solute/membrane preferential interactions are of relevance also for the membranes' short-range repulsion. ${ }^{53}$ Fig. 3A shows normalized density distributions perpendicular to the membrane surface, $\rho / \rho_{\text {cen }}$, of TMAO, urea, $\mathrm{Na}^{+}$, and $\mathrm{Cl}^{-}$ in simulations of highly hydrated POPC membranes $\left(n_{\mathrm{w}}=25\right)$ containing 10 wt\% TMAO, urea, or $\mathrm{NaCl}$, respectively. Here, $\rho_{\text {cen }}$ denotes the co-solute density at the center of the water layer. The membrane surface at $z=0$ is defined as the surface of a water layer of thickness $D_{\mathrm{w}}$ according to eqn (3).

Preferential accumulation or depletion of the respective solutes at the membrane surface is quantified in terms of the surface excess $\Gamma$, which is computed from the co-solute density profiles as

$$
\Gamma=\int_{-\infty}^{0} \rho(z) \mathrm{d} z+\int_{0}^{\infty}\left[\rho(z)-\rho_{\text {cen }}\right] \mathrm{d} z .
$$

TMAO exhibits a very strong depletion at the interface, $\Gamma_{\text {TMAO }}\left(n_{\mathrm{w}}=25\right)<0$, so that it accumulates in the center of the aqueous region. As shown in Section 3.3.5, the magnitude of this depletion is robust with respect to forcefield variations. Regarding the urea distribution, the density deficit $\left(\rho-\rho_{\text {cen }}<1\right)$ on the aqueous side $(z>0)$ is somewhat more pronounced than its excess on the membrane side $(z<0)$, reflecting a weak
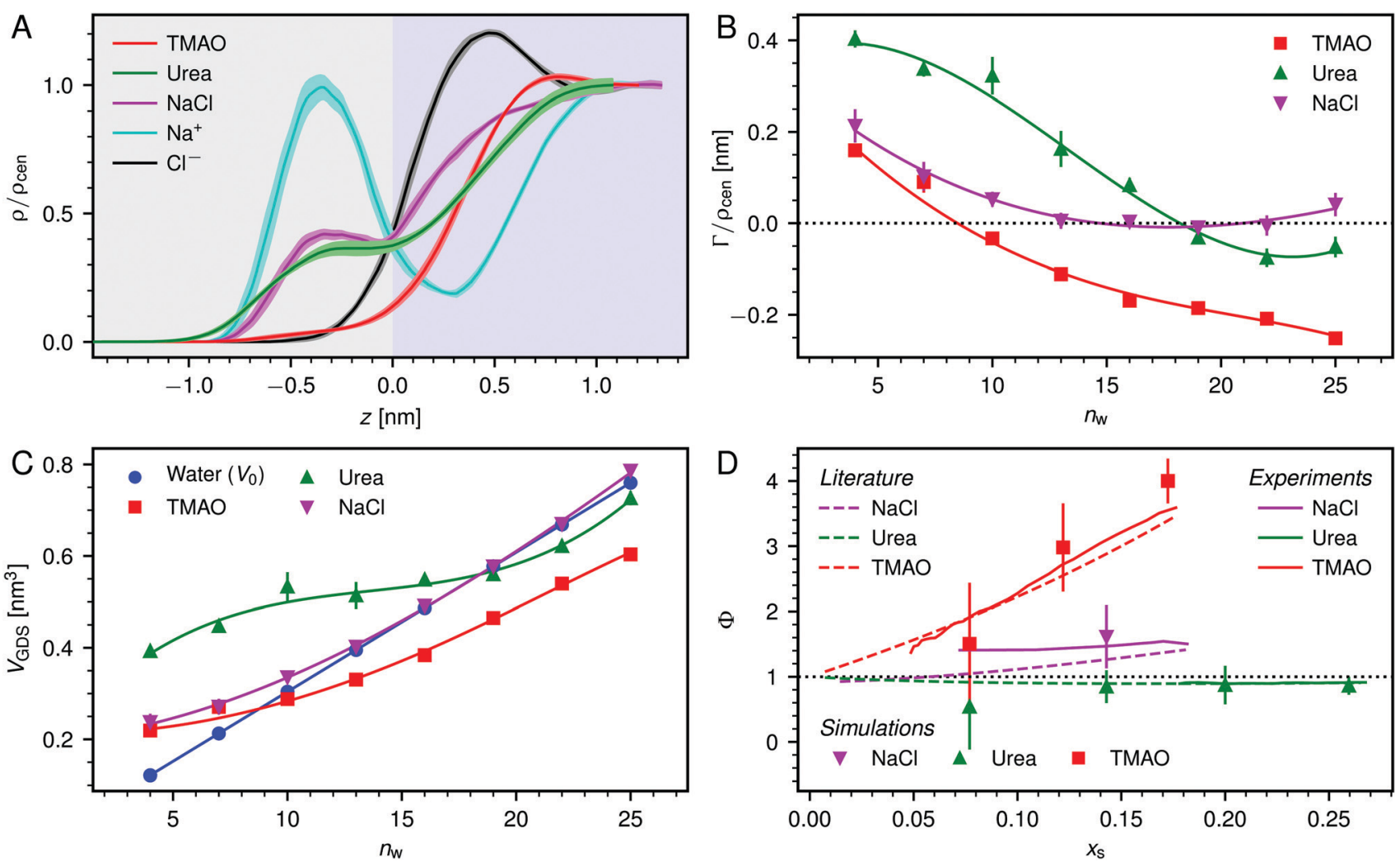

Fig. 3 (A) Normalized density profiles of the co-solutes perpendicular to the membrane surface at high hydration (POPC, $\left.n_{w}=25\right)$. The profiles are averaged over five independent simulations with $10 \mathrm{wt} \%$ of co-solutes. Shaded areas indicate standart errors of the mean. The background color indicates the location of the lipid bilayer (grey) and the water slab (purple) in the Gibbs dividing surface definition (see main text). (B) Hydration-dependent surface excess for the different co-solutes as computed from eqn (4). (C) Volume per lipid effectively accessible to the co-solutes, $V_{\mathrm{GDS}}=A_{\text {lip }} \cdot D_{\mathrm{GDS}} / 2$, as a function of the hydration level. Lines show cubic smoothing splines. (D) Concentration-dependent osmotic coefficients from the experimental literature $e^{49-51}$ and the corresponding values from sorption experiments and simulations obtained in the present work. Data are plotted over the solute mole fraction $x_{\mathrm{s}}=1-x_{\mathrm{w}}$ for clarity. The dotted horizontal line indicates ideal behavior $(\Phi=1)$. 
yet significant depletion of urea at the membrane surface at this hydration level, $\Gamma_{\text {urea }}\left(n_{\mathrm{w}}=25\right)<0$. The exact value of this depletion is however forcefield-dependent (see Section 3.3.5). In the case of $\mathrm{NaCl}$, the combined distribution of $\mathrm{Na}^{+}$and $\mathrm{Cl}^{-}$ exhibits an excess on the membrane side overcompensating the slight depletion on the aqueous side, such that the overall surface excess of $\mathrm{NaCl}$ becomes positive. The pronounced adsorption of $\mathrm{Na}^{+}$(see its individual distribution in Fig. 3A) into the headgroup region, as reported earlier, ${ }^{54}$ has however been critically discussed and attributed to shortcomings of the Berger forcefield. ${ }^{55}$ As shown in Section 3.3.5, we indeed find a strong forcefield dependence regarding this aspect.

The hydration-dependent values of $\Gamma$, normalized by $\rho_{\text {cen }}$, are shown in Fig. 3B. TMAO is strongly repelled from the membrane surface for almost all hydration levels. Urea is weakly repelled from the membrane surface at high hydration levels but attracted at low hydration. The surface excess of $\mathrm{NaCl}$ is significantly positive, especially at low hydration levels, where we stress again that this result is specific to the Berger forcefield.

The volume per lipid effectively accessible to the co-solutes is $V_{\mathrm{GDS}}=A_{\text {lip }} D_{\mathrm{GDS}} / 2$, where $D_{\mathrm{GDS}}=D_{\mathrm{w}}-2 z_{\mathrm{GDS}}$. The Gibbs dividing surface for the co-solutes, $z_{\mathrm{GDS}}$, is defined as the $z$-position for which $\Gamma$ would vanish and is computed as

$$
z_{\mathrm{GDS}}=z_{\text {lip }}+\int_{z_{\text {lip }}}^{z_{\mathrm{w}}}\left[1-\frac{\rho(z)}{\rho_{\text {cen }}}\right] \mathrm{d} z,
$$

where $z_{\text {lip }}$ and $z_{\mathrm{w}}$ denote the centers of the bilayer and of the water slab, respectively. Fig. 3C shows $V_{\mathrm{GDS}}$ as a function of $n_{\mathrm{w}}$ for all three co-solutes. It is seen that $V_{\mathrm{GDS}}^{\mathrm{TMAO}}$ is considerably smaller than the water volume $V_{0}=A_{\text {lip }} D_{\mathrm{w}} / 2=n_{\mathrm{w}} v_{\mathrm{w}}^{0}$ in almost the entire hydration range. Urea exhibits more complex behavior, with $V_{\mathrm{GDS}}^{\mathrm{urea}}>V_{0}$ at most hydration levels and $V_{\mathrm{GDS}}^{\mathrm{urea}} \lesssim V_{0}$ at very high hydration. Except for very low hydration levels, $V_{\mathrm{GDS}}^{\mathrm{NaCl}}$ remains almost equal to $V_{0}$ due to a compensation of the increasing surface excess and the decreasing area per lipid upon dehydration (see Fig. 4B), noting once more that this result is specific to the Berger forcefield.

3.3.2 Area per lipid. The area per lipid in multilamellar membrane systems is known to be affected by the hydration level. ${ }^{1,48}$ Fig. 4A shows experimental data on $A_{\text {lip }}$ for DMPC as a function of $n_{\mathrm{w}} \cdot{ }^{48}$ Our simulations show agreement with the experimental data within about $10 \%$ and also reproduce the observed decrease of the lipid area upon dehydration. Remaining deviations must be attributed to forcefield limitations and possible systematic errors introduced in the experimental procedures. For POPC, the agreement between experiments ${ }^{52}$ and simulations is similar (see Fig. 4B).

Fig. $4 \mathrm{~B}$ shows the hydration dependence of $A_{\text {lip }}$ in presence of co-solutes as obtained in the simulations with POPC bilayers. It is seen that TMAO significantly decreases $A_{\text {lip }}$, by about 2 to $3 \AA^{2}$, while urea increases $A_{\text {lip }}$ by a comparable increment, depending on the hydration level. $\mathrm{NaCl}$ has the strongest effect. It decreases $A_{\text {lip }}$ by about 7 to $8 \AA^{2}$ for all hydration levels. A simple Gibbs adsorption model, associating a positive surface excess with an increase in $A_{\text {lip }}$ and a negative surface excess with its decrease, qualitatively predicts the effect of TMAO and urea at most hydration levels. This model however neglects co-solute-induced variations in the lipid chemical potential and is thus of limited predictive power. In fact, it fails to predict the NaCl-induced decrease of $A_{\text {lip }}$, which is in line with earlier indirect experimental evidence ${ }^{55,56}$ and can be attributed to a condensation due to local charge compensation. ${ }^{54,57}$

3.3.3 Modeling co-solute effects on hydration repulsion. As shown in our earlier work, ${ }^{23}$ urea and TMAO are confined in the aqueous layer between the membrane surfaces in a wide range of osmotic pressures. This scenario is imposed by construction in the MD simulations in the present work and found to reproduce the experimental data (see above). Dehydration of the co-solute-loaded membranes is thus associated with an
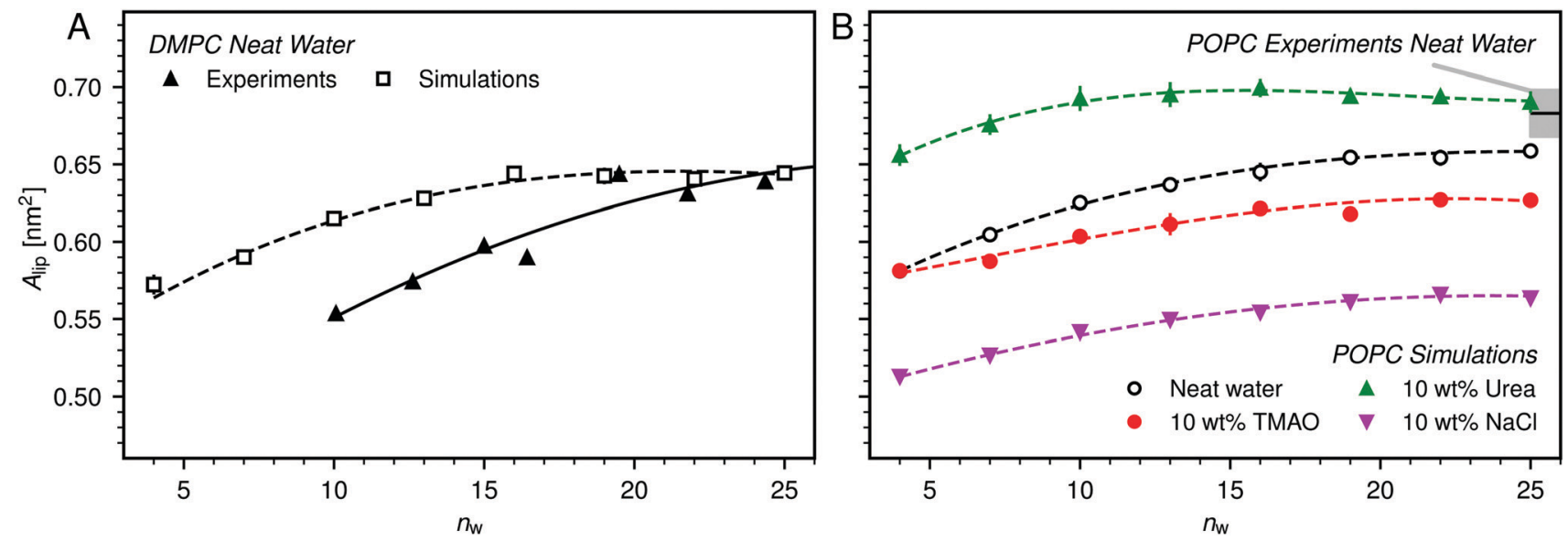

Fig. 4 (A) Average area per lipid, $A_{\text {lip, }}$ in DMPC membranes without added co-solutes as a function of the hydration level, as reported in the experimental literature $\mathrm{e}^{48}$ and as obtained from the simulations in the present work. (B) Influence of 10 wt\% TMAO, urea, or $\mathrm{NaCl}$ on $A_{\text {lip }}$ as observed in simulations of POPC bilayers. Experimental data for fully hydrated POPC bilayers in neat water is shown for comparison, with the shaded area indicating the experimental uncertainty. ${ }^{52}$ Lines are guides to the eye. 
increase in the local co-solute concentration in the water-slab, which in turn exerts a repulsive pressure $\Delta \Pi$ contributing to the surface interaction. Within an ideal mixing approximation this repulsive pressure is given by van't Hoff's law

$$
\Delta \Pi_{\text {ideal }}\left(n_{\mathrm{w}}\right)=k_{\mathrm{B}} T \frac{n_{\mathrm{cosol}}}{V_{0}\left(n_{\mathrm{w}}\right)}
$$

where $n_{\text {cosol }}$ is the number of co-solute molecules per lipid and $V_{0}=n_{\mathrm{w}} v_{\mathrm{w}}^{0}$ the associated solvent volume. The overall repulsive pressure $\Pi_{\text {ideal }}^{\text {theo }}=\Pi_{\text {water }}+\Delta \Pi_{\text {ideal }}$, is indicated with dotted lines in Fig. 5 , where $\Pi_{\text {water }}$ is an exponential fit to the hydration repulsion simulation data in pure water for $n_{\mathrm{w}}<19$. The obtained decay lengths are $\lambda_{\text {DMPC }}=0.29 \mathrm{~nm}$ and $\lambda_{\text {POPC }}=0.25 \mathrm{~nm}$, respectively, in reasonable agreement with the experimental results presented above. It is also seen that $\Pi_{\text {ideal }}^{\text {theo }}$ is in satisfactory agreement with the simulation data for membranes loaded with $10 \mathrm{wt} \%$ urea and $\mathrm{NaCl}$ but fails in the case of TMAO, where it underestimates the repulsive pressure by more than a factor of 2 .

One necessary refinement directly follows from one of our earlier conclusions: the effective volume accessible to the cosolutes, $V_{\mathrm{GDS}}$, deviates significantly from that of the solvent, $V_{0}$, meaning that eqn (6) needs to be augmented by a correction factor $V_{0} / V_{\mathrm{GDS}}$. Another refinement concerns the co-solute osmotic coefficients, $\Phi$, which substantially deviate from the ideal behavior $(\Phi=1)$ assumed in eqn (6). Fig. 3D shows osmotic coefficients of urea, TMAO, and $\mathrm{NaCl}$ in a wide concentration range, determined in independent simulations and experiments as described in the Methods section. The data are in good agreement with experimental data from the literature for urea, ${ }^{50}$ TMAO, ${ }^{49}$ and $\mathrm{NaCl}^{51}$ indicated in the figure with lines. Literature values were either computed from the reported water activity using eqn (1) or the given relation between the osmotic coefficients and the solute molality $b\left(x_{\mathrm{w}}\right)$ was used directly (for details see ESI $\dagger$ ). The solution's osmotic coefficient and the more commonly used co-solute's activity coefficient $\gamma$, are related through the Gibbs-Duhem equation as $\ln (\gamma)=\Phi-1+\int_{0}^{b}(\Phi-1) / b^{\prime} \mathrm{d} b^{\prime}$ where $b$ is the molal concentration. The agreement between simulations and experimental data for TMAO and urea in Fig. 3D is expected because the simulation forcefields have been developed with the aim to reproduce osmotic coefficients. ${ }^{34,35}$ The investigated co-solutes display different deviations from ideality: $\Phi_{\text {TMAO }}$ deviates the strongest, enhancing its osmolyte-capabilities, and $\Phi_{\mathrm{NaCl}}$ is slightly larger than unity which is typical for salts. Finally, urea forms near-ideal solutions in water.
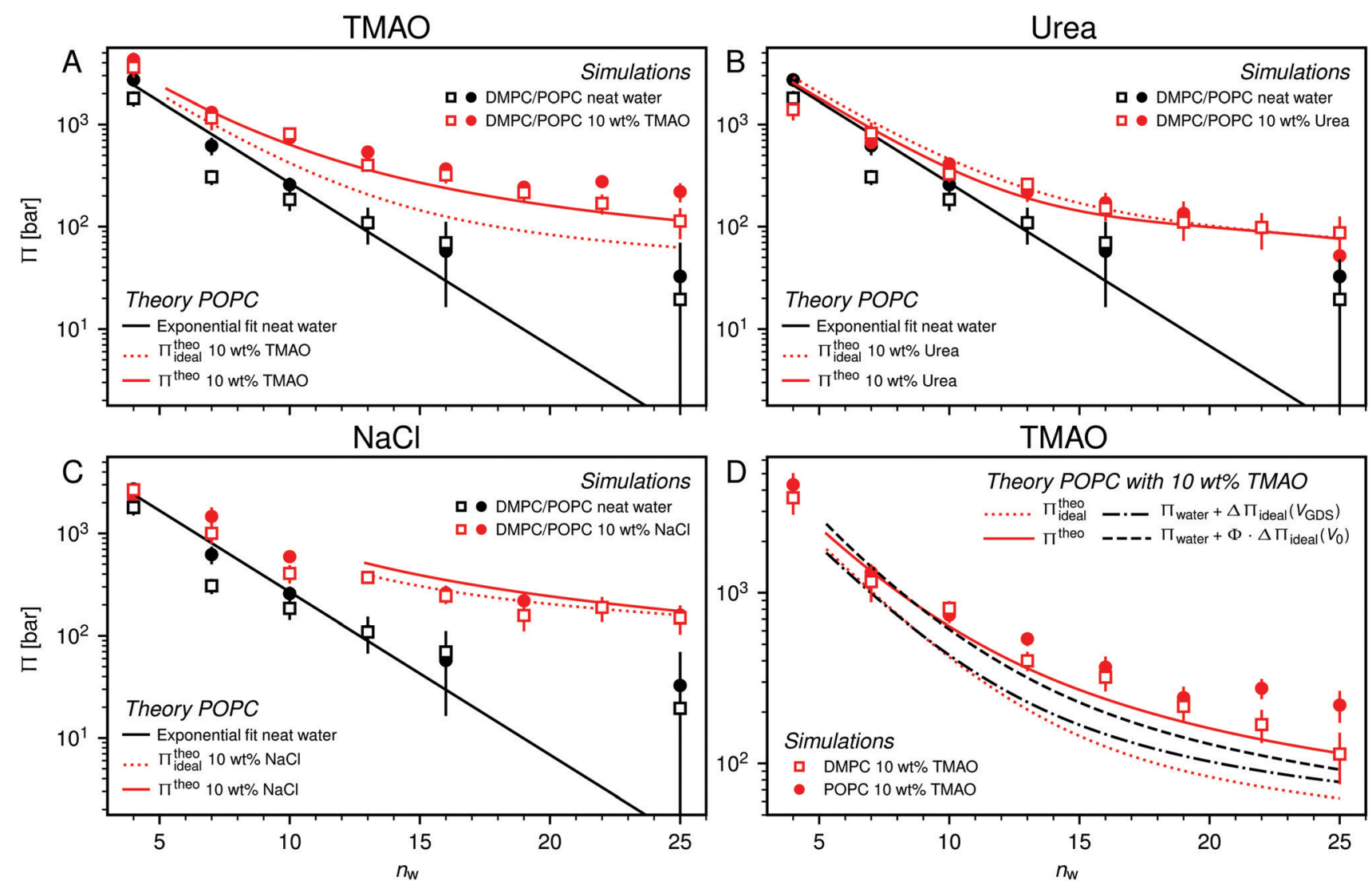

Fig. 5 Pressure-hydration curves $\Pi\left(n_{w}\right)$ of PC membranes with and without added 10 wt\% TMAO (panel A), urea (panel B), and NaCl (panel C) as obtained in the simulations. Black solid lines represent exponential fits to the simulation data for POPC without added co-solutes. Dotted and solid red lines indicate the predictions of theoretical models for POPC simulations, neglecting (eqn (6)) and accounting (eqn (7)) for effective volume and osmotic coefficient effects, respectively. Theoretical predictions are only shown for hydration levels for which the co-solute concentration is below the solubility limit. Panel (D) shows the influence of the effective volume and of the osmotic coefficient individually. 
Augmentation of eqn (6) by the co-solute osmotic coefficients and their accessible volume yields

$$
\Delta \Pi\left(n_{\mathrm{w}}\right)=\Phi\left(n_{\mathrm{w}}\right) k_{\mathrm{B}} T \frac{n_{\mathrm{cosol}}}{V_{\mathrm{GDS}}\left(n_{\mathrm{w}}\right)} .
$$

Cubic splines were used to interpolate between data points for $V_{\mathrm{GDS}}$ in order to make eqn (7) continuous over the entire hydration range. For the osmotic coefficients $\Phi\left(n_{\mathrm{w}}\right)$, we used the expressions reported in the literature. ${ }^{49-51}$

With these refinements, the theoretically predicted osmotic pressures $\Pi_{\text {theo }}=\Pi_{\text {water }}+\Delta \Pi$ fit well to the simulation data (see Fig. 5). Remaining deviations likely originate from the neglect of other effects such as additional electrostatic repulsion in the case of $\mathrm{NaCl}^{58}$ or other altered contributions to the perpendicular equation of state. ${ }^{59}$ Overall, the agreement between eqn (7) and the simulation data demonstrates that the cosolutes' osmotic pressure is at the heart of the increase in the repulsion. Closer inspection (Fig. 5D) reveals that the osmotic coefficient is the dominant correction at low hydration, while excluded-volume and osmotic coefficient effects are equally important at higher hydration. It becomes clear that TMAO leads to the strongest repulsion because it exhibits unfavorable interactions with the membrane surfaces and at the same time a remarkably high osmotic coefficient. TMAO and the lipids' PC headgroups both have a dipolar/hydrophobic architecture. The high osmotic coefficient and the pronounced depletion at the membrane surfaces therefore likely have the same physical origin previously termed dipolar/hydrophobic frustration. ${ }^{34}$

3.3.4 Repulsion thermodynamics. The free energy of membrane dehydration, $G\left(n_{\mathrm{w}}\right)$ (see Methods section), can be decomposed into its enthalpic and entropic contributions, $H\left(n_{\mathrm{w}}\right)$ and $-T S\left(n_{\mathrm{w}}\right)$, respectively, as was done earlier in experiments ${ }^{29}$ and simulations ${ }^{34}$ with satisfactory agreement. In the following, the influence of co-solutes on these individual contributions is discussed.

Fig. 6A shows the enthalpy/entropy decomposition for DMPC membranes interacting across neat water, where the methodology described earlier ${ }^{25}$ was used. It is seen that the repulsion is of entropic origin at larger hydration but crosses over to an enthalpy-driven regime at low hydration. The addition of co-solutes significantly increases the magnitude of both contributions as well as their antagonistic interplay. Fig. 6B exemplarily shows the enthalpy/entropy decomposition of the free energy associated with the dehydration of membranes
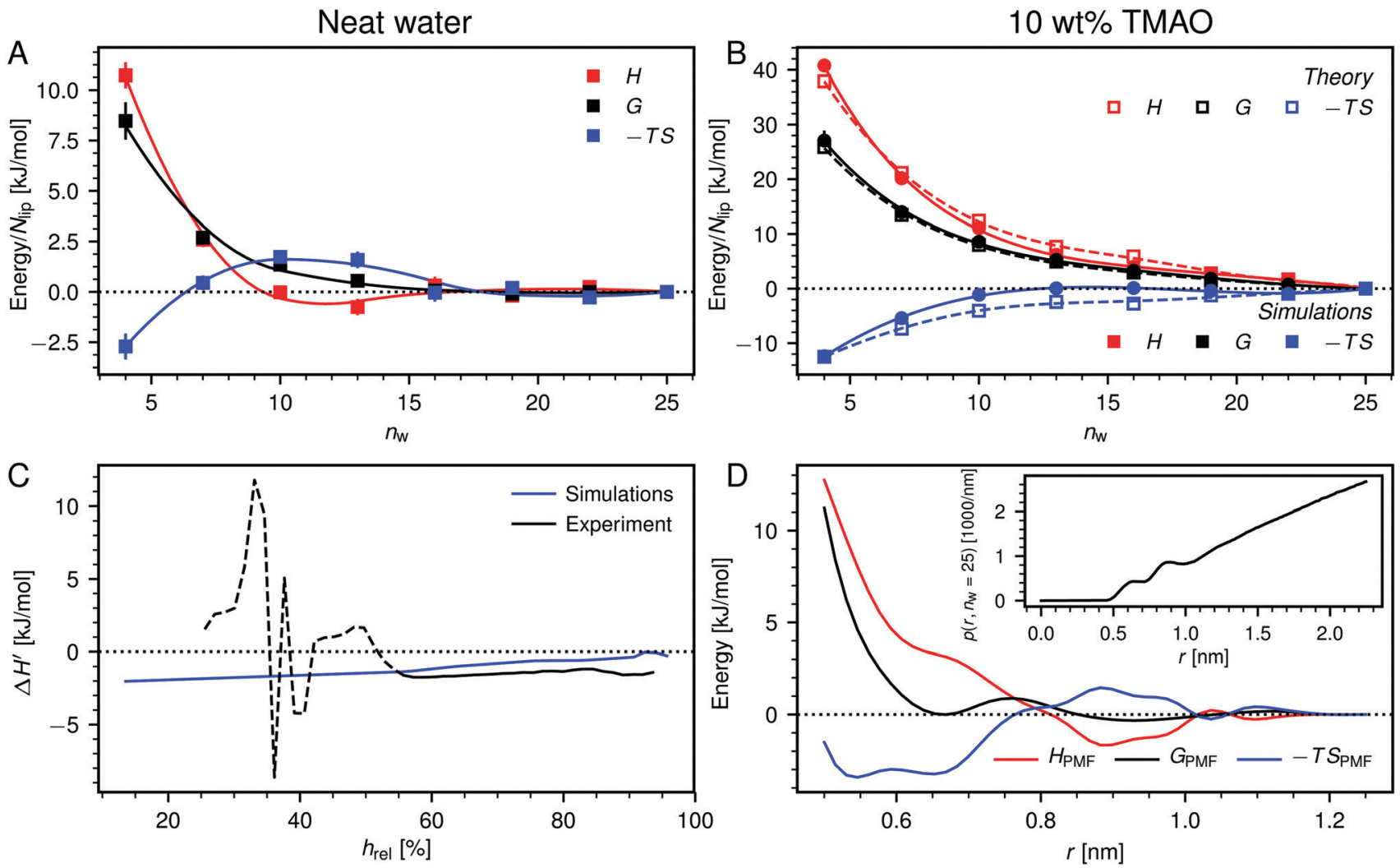

Fig. 6 (A) Decomposition of the dehydration free energy, $G\left(n_{\mathrm{w}}\right)$, in DMPC membrane simulations without added co-solutes into enthalpic and entropic contributions, $H\left(n_{w}\right)$ and $-T S\left(n_{w}\right)$, respectively. Lines are guides to the eye. (B) Same decomposition for DMPC membranes loaded with 10 wt\% TMAO. Open squares are theoretical predictions according to eqn (8). (C) Comparison between experiments and simulations of POPC lipids, regarding the change $\Delta H^{\prime}$ in the differential hydration enthalpy $H^{\prime}=\mathrm{d} H / \mathrm{d} n_{\mathrm{w}}$ due to addition of $5 \mathrm{wt} \%$ TMAO. The comparison is only valid for humidities above $h_{\mathrm{rel}} \approx 55 \%$ (indicated with a solid line style). (D) Potential of mean force, $G_{\mathrm{PMF}}(r)$, for the TMAO-TMAO interaction in bulk solutions and its enthalpic and entropic contributions, $H_{\text {PMF }}(r)$ and $-T S_{\text {PMF }}(r)$, respectively. The TMAO-TMAO center-to-center radial distance is denoted with $r$. Data are reproduced from the literature. ${ }^{34}$ Inset: Normalized TMAO-TMAO distance distribution $p\left(r, n_{\mathrm{w}}\right)$ in DMPC membranes loaded with 10 wt $\%$ TMAO at $n_{\mathrm{w}}=25$. 
loaded with 10 wt\% TMAO, for which the effect is most pronounced and for which complementary thermodynamic information is available. ${ }^{34}$ The repulsion is seen to be enthalpic throughout the entire separation range. The entropic contribution is of opposite sign but sub-dominant.

In Fig. 6C we compare experimental and simulation data for the change $\Delta H^{\prime}=H_{5 \mathrm{wt} \% \mathrm{TMAO}}^{\prime}-H_{\text {water }}^{\prime}$ in the differential enthalpy of hydration $H^{\prime}=\mathrm{d} H / \mathrm{d} n_{\mathrm{w}}$ when loading POPC membranes with 5 wt\% TMAO. The comparison is valid only for relative humidities above $h_{\text {rel }} \approx 55 \%$, because in the humidity range below that threshold (indicated with a dashed line style), TMAO forms a separate phase and POPC undergoes a phase transition in the experiments. In the meaningful humidity range (indicated with a solid line style), the simulation data semiquantitatively agree with the experiments. In fact, the agreement looks even better when $\Delta H^{\prime}$ is plotted as a function of the hydration level $n_{\mathrm{w}}$ (see Fig. S5 in the ESI $\dagger$ ). One should keep in mind, however, that in this representation, the water chemical potential deviates between experiments and simulations.

The pairwise mutual repulsion between TMAO molecules in water was reported to be of enthalpic origin ${ }^{34}$ (see Fig. 6D) and the dehydration of TMAO-loaded membranes is associated with a reduction in the average TMAO/TMAO distance. The enthalpic character of the repulsion between TMAO-loaded membranes can therefore be attributed to the enthalpic character of the TMAO/TMAO mutual repulsion. In fact, the interaction thermodynamics appear dominated by the dehydration of the confined TMAO solution. To take this concept to a quantitative level, the dehydration thermodynamics of TMAO-loaded lipid membranes is approximated in the following as a linear combination of the dehydration thermodynamics of pure membrane dehydration $\left(G_{\text {lip }}^{\text {theo }}, H_{\text {lip }}^{\text {theo }}\right.$, and $\left.S_{\text {lip }}^{\text {theo }}\right)$ and of the thermodynamics of the TMAO/ TMAO pairwise interaction $\left(G_{\text {TMAO }}^{\text {theo }}, H_{\text {TMAO }}^{\text {theo }}\right.$ and $\left.S_{\text {TMAO }}^{\text {theo }}\right)$,

$$
\begin{aligned}
& G^{\text {theo }}\left(n_{\mathrm{w}}\right)=G_{\text {lip }}^{\text {theo }}\left(n_{\mathrm{w}}\right)+K \cdot G_{\text {TMAO }}^{\text {theo }}\left(n_{\mathrm{w}}\right) \\
& H^{\text {theo }}\left(n_{\mathrm{w}}\right)=H_{\text {lip }}^{\text {theo }}\left(n_{\mathrm{w}}\right)+K \cdot H_{\text {TMAO }}^{\text {theo }}\left(n_{\mathrm{w}}\right) \\
& S^{\text {theo }}\left(n_{\mathrm{w}}\right)=S_{\text {lip }}^{\text {theo }}\left(n_{\mathrm{w}}\right)+K \cdot S_{\text {TMAO }}^{\text {theo }}\left(n_{\mathrm{w}}\right),
\end{aligned}
$$

where $K$ is a weighting coefficient discussed further below. The contributions of the TMAO/TMAO pairwise interaction were computed by averaging $H_{\mathrm{PMF}}(r), G_{\mathrm{PMF}}(r)$, and $S_{\mathrm{PMF}}(r)$ in Fig. 6D over the hydration-dependent distribution $p\left(r, n_{w}\right)$ of TMAOTMAO pair distances $r$ (for details see Fig. S6 in the ESI $\dagger$ ),

$$
\begin{aligned}
& G_{\mathrm{TMAO}}^{\text {theo }}\left(n_{\mathrm{w}}\right)=\int_{0}^{\infty} G_{\mathrm{PMF}}(r) \cdot p\left(r, n_{\mathrm{w}}\right) \mathrm{d} r \\
& H_{\mathrm{TMAO}}^{\text {theo }}\left(n_{\mathrm{w}}\right)=\int_{0}^{\infty} H_{\mathrm{PMF}}(r) \cdot p\left(r, n_{\mathrm{w}}\right) \mathrm{d} r \\
& S_{\mathrm{TMAO}}^{\text {theo }}\left(n_{\mathrm{w}}\right)=\int_{0}^{\infty} S_{\mathrm{PMF}}(r) \cdot p\left(r, n_{\mathrm{w}}\right) \mathrm{d} r .
\end{aligned}
$$

The inset of Fig. 6D exemplarily shows $p(r)$ for $n_{\mathrm{w}}=25$. The distribution is normalized such that it amounts to the number of TMAO-TMAO pairwise interactions in the simulated system, $N_{\text {TMAO }}^{\text {pair }}$,

$$
\int_{0}^{\infty} p\left(r, n_{\mathrm{w}}\right) \mathrm{d} r=N_{\mathrm{TMAO}}^{\text {pair }}=\frac{N_{\mathrm{TMAO}}\left(N_{\mathrm{TMAO}}-1\right)}{2} .
$$

Open symbols in Fig. 6B indicate the predictions of eqn (8) after adjusting the weighting coefficient $K$ to match the simulation data. The simultaneous satisfactory agreement of free energy, enthalpy, and entropy for a single coefficient suggests that the thermodynamics of the dehydration of membranes loaded with co-solutes can indeed be approximated as a linear combination of membrane dehydration in the absence of co-solutes and the co-solute pair-wise interaction. The best-matching weighting coefficients $\left(K_{\mathrm{POPC}}=2.23\right.$ and $K_{\mathrm{DMPC}}=2.29$ ), however, significantly deviate from unity. This deviation can be attributed to the following shortcomings of eqn (8). Firstly, the interaction between TMAO molecules in concentrated solutions is a multi-body problem and deviates from the sum of pair-wise interactions. And second, eqn (8) neglect interactions between TMAO and the PC headgroups of the lipids. These interactions are likely of similar nature as the TMAO/TMAO interactions, due to the dipolar/hydrophobic architecture of both TMAO and $\mathrm{PC}$, and also comparable in number. Indeed, analysis of the temperature-dependence of the TMAO-membrane potential of mean force (PMF, see Fig. S7 in the ESI $\dagger$ ) indicates that the repulsion between TMAO and the membrane surface is driven by enthalpy. Such enthalpy-driven exclusion of uncharged solutes from macromolecular surfaces was earlier found to be important also for the stabilization of proteins by osmolytes. ${ }^{60}$

3.3.5 Influence of the simulation forcefields. It has previously been suggested that the Berger lipid forcefield exhibits unrealistically high affinity for $\mathrm{Na}^{+}$ions. ${ }^{55}$ Indirect comparison to experimental data by means of the salt-dependent head group order parameter suggests that the CHARMM36 lipid forcefield reproduces the salt adsorption more realistically. ${ }^{55}$ In order to evaluate the robustness of the present simulation results with respect to the choice of the forcefields, we performed additional simulations employing the CHARMM36 forcefield for the lipids and $\mathrm{NaCl}$ in combination with the TIP3P water model. Fig. 7A shows a comparison of the $\mathrm{NaCl}$ density profiles at the POPC interface for the Berger-based versus the CHARMM36based forcefield combinations. Indeed, the $\mathrm{Na}^{+}$adsorption and thus the surface excess of $\mathrm{NaCl}$ (Fig. 7B) and the accessible volume (Fig. 7C) are significantly higher for the Berger-based forcefield combination. Additional simulations involving variation of the ion forcefield for each lipid forcefield confirm that the lipid forcefield is determining the strength of the $\mathrm{Na}^{+}$-adsorption (see Fig. S8 in the ESI $\dagger$ ).

Fig. 7D shows a comparison between experimental pressuredistance curves (POPC loaded with $5 \mathrm{wt} \% \mathrm{NaCl}$ ) and those predicted by the Berger-based and CHARMM36-based forcefield combinations. It is apparent that, especially at larger membrane separations, the added repulsion due to the presence of $\mathrm{NaCl}$ is better captured by the CHARMM36-based forcefield combination. This difference can be partially attributed to the difference in $V_{\mathrm{GDS}}^{\mathrm{NaCl}}$ (see Fig. 7C and eqn (7)). It should be noted, however, that the bulk activity coefficient of the $\mathrm{NaCl}$ forcefield used in the CHARMM36-based forcefield combination deviates significantly from the experimental behavior (see Fig. S9 in the ESI $\dagger$ ). So the CHARMM36 results in Fig. 7 should be considered carefully.

Selected simulations with a CHARMM36-based forcefield combination (i.e., with the CHARMM36 lipid forcefield and 
A
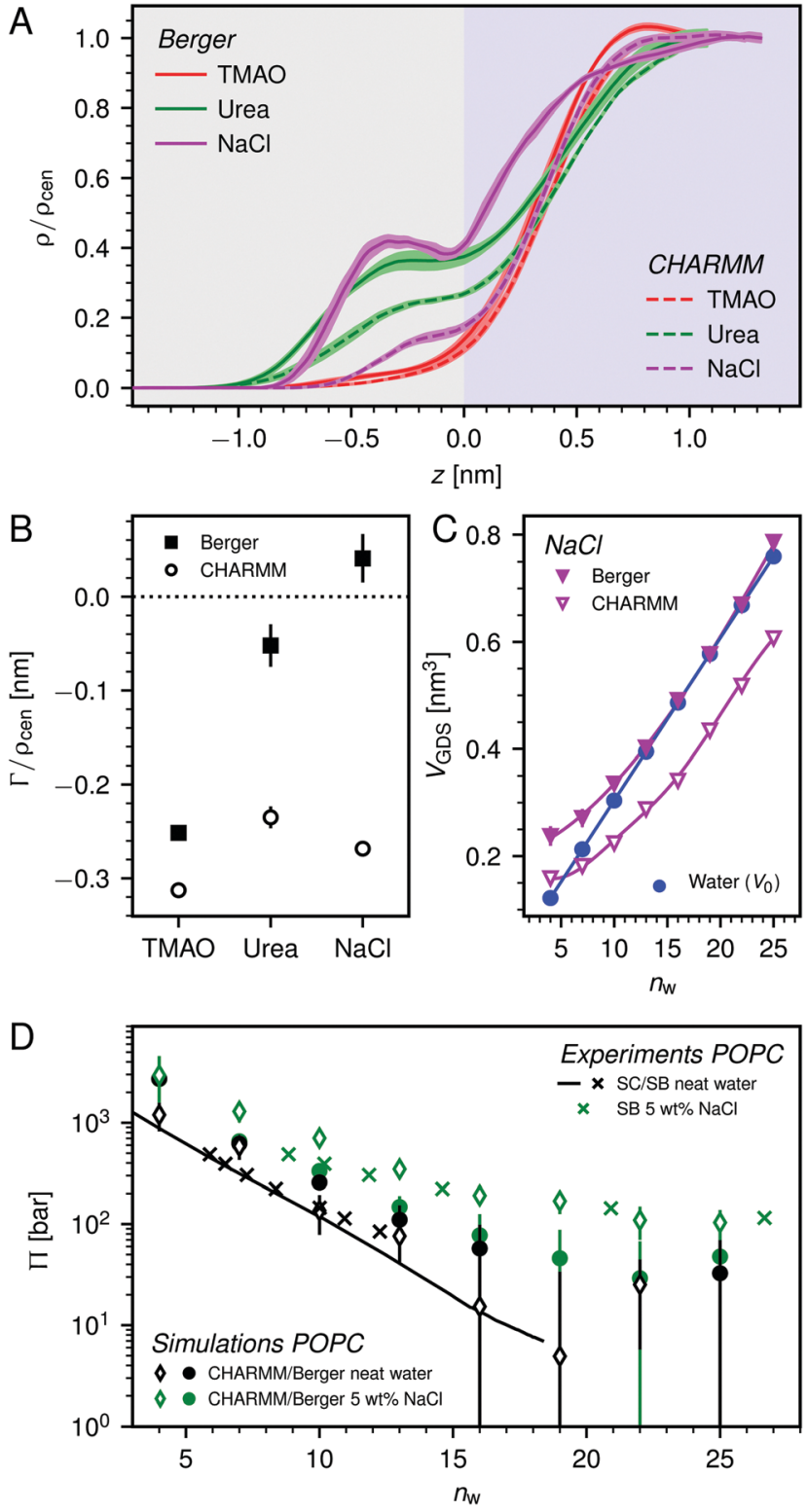

Fig. 7 Analysis of the influence of the lipid forcefield. (A) Density profiles of the respective co-solutes at the POPC lipid interface at high hydration $\left(n_{\mathrm{w}}=25\right)$. (B) Co-solute surface excess at $n_{\mathrm{w}}=25$ as obtained with both forcefield combinations. (C) Hydration dependent accessible volume from $\mathrm{NaCl}$ simulations at the POPC interface for both forcefield combinations. The neat water volume $V_{0}$ is shown for comparison. Lines are guides to the eye. (D) Pressure-distance curves obtained for both forcefield combinations in comparison to experimental data.

TIP3P water) were also performed for the co-solutes TMAO and urea and compared to the results obtained with the Berger-based forcefield combination. As shown in Fig. 7A and B, the influence of the forcefield is practically negligible for TMAO, the osmolyte most extensively discussed in the present work. For urea the agreement between the two forcefield combinations is only qualitative and consistent only with regard to the negative sign of the surface excess (see Fig. 7B). It is, however, important to note that irrespective of any forcefield effects on the quantitative level, all simulations reproduce the repulsion-enhancement due to the presence of co-solutes at least semi-quantitatively (see Fig. 2). More importantly, the physical insights into the repulsion mechanisms are gained from a self-consistent comparison between the simulation results and a theoretical model involving parameters determined with the same forcefields.

\section{Conclusions}

By combining sorption calorimetry and sorption balance experiments with molecular dynamics simulations, the influence of TMAO, urea, and $\mathrm{NaCl}$ on the hydration repulsion between stacked phospholipid membranes was investigated. All these co-solutes are found to enhance the repulsion. For a given level of dehydrating osmotic stress, the presence of cosolutes leads to the retention of a higher hydration level in terms of number of water molecules per lipid or per membrane area. The co-solutes therefore do not only substitute for water molecules but additionally have a moisturizing effect in the literal sense. The simulations, which accurately yield the chemical potential of water, quantitatively reproduce the experimental data and provide detailed insight into the mechanism by which the co-solutes enhance the hydration repulsion.

The osmotic pressure generated by the co-solutes confined in the aqueous layer between the membrane surfaces is identified as the source of the additional repulsion. The strength of this effect is found to be strongly dependent on the preferential interaction of the co-solutes with the membrane surfaces and on the osmotic coefficient of the confined co-solute solution. For high enough co-solute contents, the thermodynamics of membrane dehydration is dominated by the thermodynamics of the dehydration of the co-solute solution. With that, the simulations provide the explanation for the remarkably strong moisturizing effect of the osmolyte TMAO, which exhibits highly unfavorable interactions with the membrane surfaces and at the same time a high osmotic coefficient.

In an earlier study by Sukenik et al., ${ }^{24}$ TMAO was reported to dehydrate bilayer systems under excess water conditions when TMAO is in a phase-separated aqueous phase. Here we show that the trend is reversed in the absence of excess water, which is the relevant boundary condition in the stratum corneum. This complete reversal of the effect of a co-solute depending on the imposed boundary conditions demonstrates that the same type of co-solute can be used by nature for both moisturizing and dehydrating purposes. In fact, this reversal can be identified also in the data reported by Sukenik et al., where TMAO enhances the hydration repulsion at the highest applied osmotic pressures. Based on the insights gained in our present work, this observation suggests that under these conditions TMAO partitions at least partially into the lipid multilayers, where it contributes to the repulsion via the same mechanisms described above.

\section{Conflicts of interest}

There are no conflicts to declare. 


\section{Acknowledgements}

E. Schneck acknowledges support by the Max Planck Society and by the German Research Foundation (DFG) via Emmy-Noether grant (SCHN 1396/1). The DFG is gratefully acknowledged also for financial support (R. R. N. and A. W.-K.) via grant SFB1078. E. Sparr and Q. D. P. acknowledge support by the Swedish Research Council (VR), both through regular grants and the Linnaeus Center of Excellence "Organizing molecular matter". We thank Lars Wadsö and Vitaly Kocherbitov for help with the sorption calorimetry measurements. We kindly acknowledge Håkan Wennerström for fruitful discussions. Open Access funding provided by the Max Planck Society.

\section{References}

1 V. A. Parsegian, N. Fuller and R. P. Rand, Proc. Natl. Acad. Sci. U. S. A., 1979, 76, 2750-2754.

2 J. Israelachvili and H. Wennerström, Nature, 1996, 379, 219. 3 R. Lipowsky, Nature, 1991, 349, 475.

4 J. Ulminus, H. Wennerstrom, G. Lindblom and G. Arvidson, Biochemistry, 1977, 16, 5742-5745.

5 B. Kowalik, T. Schubert, H. Wada, M. Tanaka, R. R. Netz and E. Schneck, J. Phys. Chem. B, 2015, 119, 14157-14167.

6 E. Sparr and H. Wennerström, Biophys. J., 2001, 81, 1014-1028.

7 P. M. Elias, J. Controlled Release, 1991, 15, 199-208.

8 R. Schulz, K. Yamamoto, A. Klossek, R. Flesch, S. Hönzke, F. Rancan, A. Vogt, U. Blume-Peytavi, S. Hedtrich and M. Schäfer-Korting, et al., Proc. Natl. Acad. Sci. U. S. A., 2017, 114, 3631-3636.

9 K. B. Storey and J. M. Storey, Physiol. Rev., 1988, 68, 27-84.

10 P. H. Yancey, J. Exp. Biol., 2005, 208, 2819-2830.

11 G. O. Kirst, Annu. Rev. Plant Physiol. Plant Mol. Biol., 1990, 41, 21-53.

12 I. J. Vereyken, V. Chupin, A. Islamov, A. Kuklin, D. K. Hincha and B. de Kruijff, Biophys. J., 2003, 85, 3058-3065.

13 K. N. Barton, M. M. Buhr and J. S. Ballantyne, Am. J. Physiol., 1999, 276, R397-R406.

14 U.-B. Ramløy, Hum. Reprod., 2000, 15, 26-46.

15 M. Hara and A. Verkman, Proc. Natl. Acad. Sci. U. S. A., 2003, 100, 7360-7365.

16 A. V. Rawlings, I. R. Scott, C. R. Harding and P. A. Bowser, J. Invest. Dermatol., 1994, 103, 731-740.

17 S. Björklund, J. M. Andersson, Q. D. Pham, A. Nowacka, D. Topgaard and E. Sparr, Soft Matter, 2014, 10, 4535-4546.

18 C. Katagiri, J. Sato, J. Nomura and M. Denda, J. Dermatol. Sci., 2003, 31, 29-35.

19 S. Kezic, G. O’Regan, N. Yau, A. Sandilands, H. Chen, L. Campbell, K. Kroboth, R. Watson, M. Rowland and W. Irwin McLean, et al., Allergy, 2011, 66, 934-940.

20 J. H. Crowe, L. M. Crowe and D. Chapman, Science, 1984, 223, 701-703.

21 F. O. Costa-Balogh, H. Wennerström, L. Wadsö and E. Sparr, J. Phys. Chem. B, 2006, 110, 23845-23852.

22 A. Nowacka, S. Douezan, L. Wadsö, D. Topgaard and E. Sparr, Soft Matter, 2012, 8, 1482-1491.
23 Q. D. Pham, A. Wolde-Kidan, A. Gupta, A. Schlaich, E. Schneck, R. R. Netz and E. Sparr, J. Phys. Chem. B, 2018, 122, 6471-6482.

24 S. Sukenik, S. Dunsky, A. Barnoy, I. Shumilin and D. Harries, Phys. Chem. Chem. Phys., 2017, 19, 29862-29871.

25 E. Schneck, F. Sedlmeier and R. R. Netz, Proc. Natl. Acad. Sci. U. S. A., 2012, 109, 14405-14409.

26 M. Kanduč, A. Schlaich, A. H. de Vries, J. Jouhet, E. Maréchal, B. Demé, R. R. Netz and E. Schneck, Nat. Commun., 2017, 8, 14899.

27 A. Nilsson, A. Holmgren and G. Lindblom, Biochemistry, 1991, 30, 2126-2133.

28 T. McIntosh, A. Magid and S. Simon, Biochemistry, 1987, 26, 7325-7332.

29 N. Markova, E. Sparr, L. Wadsö and H. Wennerström, J. Phys. Chem. B, 2000, 104, 8053-8060.

30 R. A. Robinson and R. H. Stokes, Electrolyte Solutions, Dover Publications, Incorporated, 2nd revised edition, 1959.

31 M. J. Abraham, T. Murtola, R. Schulz, S. Páll, J. C. Smith, B. Hess and E. Lindah, SoftwareX, 2015, 1-2, 19-25.

32 O. Berger, O. Edholm and F. Jähnig, Biophys. J., 1997, 72, 2002-2013.

33 H. J. C. Berendsen, J. R. Grigera and T. P. Straatsma, J. Phys. Chem., 1987, 91, 6269-6271.

34 E. Schneck, D. Horinek and R. R. Netz, J. Phys. Chem. B, 2013, 117, 8310-8321.

35 S. Weerasinghe and P. E. Smith, J. Phys. Chem. B, 2003, 107, 3891-3898.

36 L. X. Dang, J. Am. Chem. Soc., 1995, 117, 6954-6960.

37 J. B. Klauda, R. M. Venable, J. A. Freites, J. W. O’Connor, D. J. Tobias, C. Mondragon-Ramirez, I. Vorobyov, A. D. MacKerell and R. W. Pastor, J. Phys. Chem. B, 2010, 114, 7830-7843.

38 R. M. Venable, Y. Luo, K. Gawrisch, B. Roux and R. W. Pastor, J. Phys. Chem. B, 2013, 117, 10183-10192.

39 W. L. Jorgensen, J. Chandrasekhar, J. D. Madura, R. W. Impey and M. L. Klein, J. Chem. Phys., 1983, 79, 926-935.

40 S. R. Durell, B. R. Brooks and A. Ben-Naim, J. Phys. Chem., 1994, 98, 2198-2202.

41 G. Bussi, D. Donadio and M. Parrinello, J. Chem. Phys., 2007, 126, 014101.

42 H. J. C. Berendsen, J. P. M. Postma, W. F. van Gunsteren, A. DiNola and J. R. Haak, J. Chem. Phys., 1984, 81, 3684-3690.

43 T. Darden, D. York and L. Pedersen, J. Chem. Phys., 1993, 98, 10089-10092.

44 B. Widom, J. Chem. Phys., 1963, 39, 2808.

45 C. H. Bennett, J. Comput. Phys., 1976, 22, 245.

46 T.-Y. Lin and S. N. Timasheff, Biochemistry, 1994, 33, 12695-12701.

47 B. Kowalik, A. Schlaich, M. Kanduč, E. Schneck and R. R. Netz, J. Phys. Chem. Lett., 2017, 2869-2874.

48 L. J. Lis, M. McAlister, N. Fuller, R. P. Rand and V. A. Parsegian, Biophys. J., 1982, 37, 657-665.

49 E. S. Courtenay, M. W. Capp, C. F. Anderson and M. T. Record, Biochemistry, 2000, 39, 4455-4471.

50 R. H. Stokes, Aust. J. Chem., 1967, 20, 2087-2100. 
51 A. R. G. Lang, Aust. J. Chem., 1967, 20, 2017-2023.

52 N. Kučerka, S. Tristram-Nagle and J. F. Nagle, J. Membr. Biol., 2006, 208, 193-202.

53 A. Schlaich, B. Kowalik, M. Kanduč, E. Schneck and R. R. Netz, Phys. A, 2015, 418, 105-125.

54 R. A. Böckmann, A. Hac, T. Heimburg and H. Grubmüller, Biophys. J., 2003, 85, 1647-1655.

55 A. Catte, M. Girych, M. Javanainen, C. Loison, J. Melcr, M. S. Miettinen, L. Monticelli, J. Määttä, V. S. Oganesyan, O. H. S. Ollila, J. Tynkkynen and S. Vilov, Phys. Chem. Chem. Phys., 2016, 18, 32560-32569.
56 G. Pabst, A. Hodzic, J. Štrancar, S. Danner, M. Rappolt and P. Laggner, Biophys. J., 2007, 93, 2688-2696.

57 R. Vácha, S. W. Siu, M. Petrov, R. A. Böckmann, J. BaruchaKraszewska, P. Jurkiewicz, M. Hof, M. L. Berkowitz and P. Jungwirth, J. Phys. Chem. A, 2009, 113, 7235-7243.

58 H. I. Petrache, T. Zemb, L. Belloni and V. A. Parsegian, Proc. Natl. Acad. Sci. U. S. A., 2006, 103, 7982-7987.

59 E. Leontidis, A. Aroti, L. Belloni, M. Dubois and T. Zemb, Biophys. J., 2007, 93, 1591-1607.

60 L. Sapir and D. Harries, Curr. Opin. Colloid Interface Sci., 2015, 20, 3-10. 